\title{
Research on the application of uniportal video-assisted thoracoscopic segmental resection of the lung in elderly patients with non-small cell lung cancer aged over 65 years
}

\author{
Hongya Xie ${ }^{1}$, Jinxing Tang ${ }^{2}$, Donglin Zhu ${ }^{1}$, Guangda Yuan ${ }^{1}$, Tengteng Wei ${ }^{1}$, Xiaoqiang Liu $^{1}$, Yong Yang ${ }^{1}$ \\ ${ }^{1}$ Department of Thoracic Surgery, The Affiliated Suzhou Hospital of Nanjing Medical University, Suzhou, China \\ ${ }^{2}$ Department of Thoracic Surgery, Henan Provincial People's Hospital, Henan, China
}

Videosurgery Miniinv 2021; 16 (3): 571-579

DOI: https://doi.org/10.5114/wiitm.2021.106829

\begin{abstract}
Introduction: The literature regarding the application of uniportal video-assisted thoracoscopic segmental resection of the lung in patients aged over 65 years with non-small cell lung cancer (NSCLC) is sparse. This paper reports 175 cases of uniportal video-assisted thoracoscopic segmental resection of the lung performed at one center, of which 63 patients were over 65 years old.

Aim: To investigate the safety and feasibility of uniportal video-assisted thoracoscopic segmental resection of the lung in elderly patients aged over 65 years with NSCLC.

Material and methods: A retrospective analysis of 175 NSCLC patients who underwent uniport video-assisted thoracoscopic segmental resection of the lung in the center from August 2018 to August 2020 was conducted, and based on the age of 65 years, patients were divided into elderly and non-elderly groups. The general data and perioperative indicators of the two groups were compared.

Results: The procedures were completed in all patients without death or conversion to open surgery. In the general data of the two groups of patients, the prevalence of emphysema in the elderly group was significantly higher than that in the non-elderly group $(p=0.001)$. However, there was no statistically significant difference between the two groups in surgery time, intraoperative blood loss, thoracic drainage tube retention time, postoperative hospital stay, incision satisfaction, or postoperative complications ( $p>0.05)$.

Conclusions: Uniportal video-assisted thoracoscopic segmental resection of the lung is feasible and safe in elderly patients with NSCLC aged over 65 years.
\end{abstract}

Key words: uniportal, segmental resection, elderly patients, non-small cell lung cancer.

\section{Introduction}

In recent years, with the acceleration of population aging, the incidence of lung cancer in the elderly is increasing annually [1]. The elderly are often burdened by multiple organ dysfunction, underlying diseases, and low immunity, which increases the risk of complications of traditional lung cancer surgery $[2,3]$. Currently, uniportal video-assisted thoracoscopic surgery (VATS) is being increasingly used in thoracic surgery. Compared with traditional multi-portal surgery, uniportal VATS has achieved the quintessential level in minimally invasive incisions and is gradually accepted by more and more thoracic surgeons, especially young physicians [4, 5]. However, the literature regarding the application of uniportal video-assisted thoracoscopic segmental

\section{Address for correspondence}

Yong Yang PhD, Department of Thoracic Surgery, The Affiliated Suzhou Hospital of Nanjing Medical University, Nanjing, China,

E-mail: 619644790@qq.com 
resection of the lung in patients aged over 65 years with non-small cell lung cancer (NSCLC) is sparse. This paper reports 175 cases of uniportal video-assisted thoracoscopic segmental resection of the lung performed at the center, of which 63 patients were over 65 years old.

\section{Aim}

To investigate the safety and feasibility of uniportal video-assisted thoracoscopic segmental resection of the lung in elderly patients aged over 65 years with NSCLC.

\section{Material and methods}

\section{Research objects}

A retrospective analysis of 175 NSCLC patients who underwent uniport video-assisted thoracoscopic segmental resection of the lung in the center from August 2018 to August 2020 was conducted, and patients were divided into two groups according to their age. A total of 175 patients were divided into two groups according to age. Among them, 63 patients were divided into the elderly group because they were 65 years old or older, and the other 112 patients aged less than 65 were in the non-elderly group. There were 28 males and 35 females in the elderly group, and 44 males and 68 females in the non-elderly group.

\section{Ethics statement}

The study was conducted in accordance with the principles of the Declaration of Helsinki, and the study protocol was approved by the ethics committee of our hospital (register number: 2020(036)). All patients signed informed consent and were informed that their data would be used for study purposes.

\section{Inclusion criteria}

(1) Preoperative computed tomography (CT) revealed pure ground-glass nodules (GGN) or mixed ground-glass nodules (mixed GGN), and the solid component of mixed GGN was $<50 \%$. (2) Tumor size was $<2 \mathrm{~cm}$, and the postoperative staging was T1NOMO peripheral NSCLC. (3) Wedge resection could not be performed or wedge resection margin distance was not $>2 \mathrm{~cm}$ for GGN.

\section{Surgical methods}

Conventional preoperative ZiWeiDiXing interactive medical image control system (ZWDX, China) was used to convert two-dimensional (2D) lung CT images into three-dimensional (3D) images, and the bronchi and blood vessels in the lung segment where pulmonary nodules were located were studied for the best surgical planning (Photo 1). Under intravenous-inhalation combined anesthesia, the patients were intubated with a double-lumen endotracheal tube and maintained in the $90^{\circ}$ decubitus position on the healthy side. In the $4^{\text {th }}$ or $5^{\text {th }}$ intercostal space, a single incision with the length of $3 \mathrm{~cm}$ was made between the anterior axillary line and midaxillary line. The incision protective cover, the video camera of the thoracoscopy, and all surgical instruments were placed in the incision. First, the condition of the diseased lung was examined to determine the location of the pulmonary nodules and lung segment to be resected. According to the preoperative 3D images, the target segmental pulmonary artery, segmental pulmonary vein and bronchus were dissociated and processed. To determine the boundary between the lung segments, the inflation-deflation method was used for the majority of patients, and the anesthesiologist was asked to use pure oxygen low pressure ventilation to expand the lung. The boundaries between segments could be displayed after about 10 min (Photos $2 \mathrm{~A}, \mathrm{~B}$ ). According to the $\mathrm{CT}$ findings, the indocyanine green (ICG) reverse staining method was used to determine the boundary between the lung segments for patients preoperatively diagnosed with emphysema. At this time, the thoracoscopy system was changed to fluorescence mode, and patients were injected with $25 \mathrm{mg}$ of ICG via the peripheral vein. After ICG reached the lung tissue through the pulmonary artery, the target segment to be resected did not display color, whereas the remaining lung tissue showed green color, forming a clear boundary on the surface of the lung. Electrocautery was used to label the intersegmental plane, and then the thoracoscopy exited fluorescence mode (Photos 2 C, D). Finally, the target lung segment was resected. An endoscopic stapler can be used to deal with the intersegmental fissure, segmental bronchus, and thicker segmental pulmonary vessels. Ultrasonic knife, ham-o-loc, or silk thread ligation can be used for the pulmonary arteriole. After rapid frozen pathology of the resected lesion, the mediastinal lymph nodes should only be sampled 
A

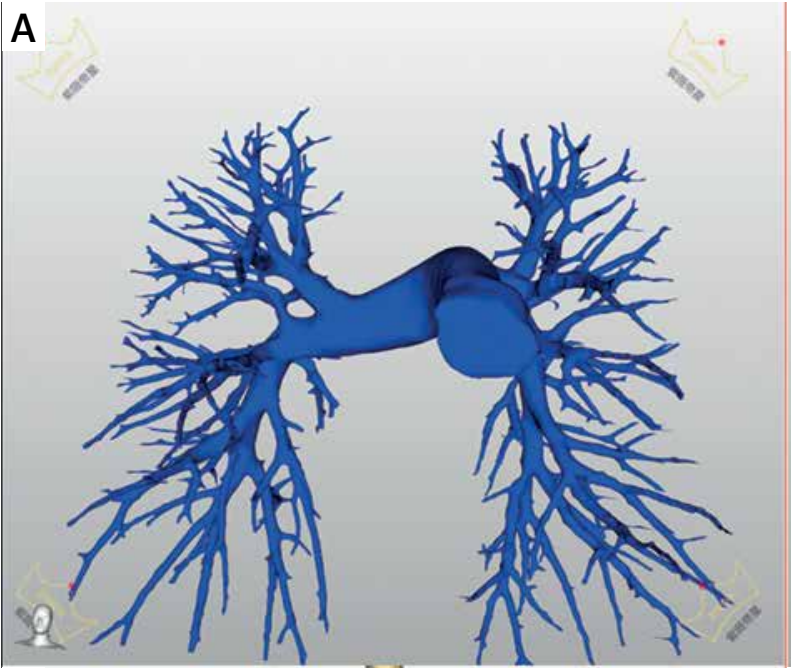

c
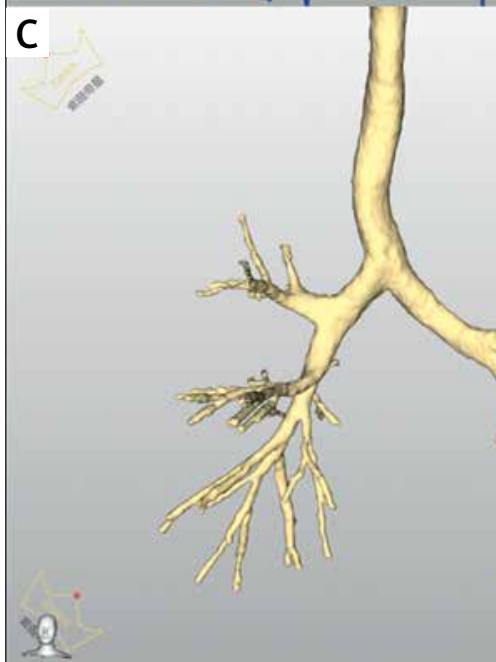

Photo 1. Converting 2D image to 3D image. A - 3D image of pulmonary artery. B - 3D image of pulmonary vein. $\mathbf{C}-3 \mathrm{D}$ image of pulmonary bronchus. $\mathrm{D}-3 \mathrm{D}$ image of target lung to be resected

when the result shows adenocarcinoma in situ or microinfiltrative adenocarcinoma, or mediastinal lymph node dissection is required systematically when the result shows infiltrative adenocarcinoma. In general, the lymph nodes in groups 4-12 were dissected in the left lung, while those in groups 2, 3, 4, 7-12 were dissected in the right lung, and the recurrent laryngeal nerve was protected. When suturing the incision, two sutures were drawn from the sutured muscle layer to fix the thoracic drainage tube. The fishbone suture of Johnson \& Johnson was used for the intradermal suturing of the skin layer.

\section{Statistical analysis}

Continuous data were expressed as the mean \pm standard deviation (SD). Significance between the
B
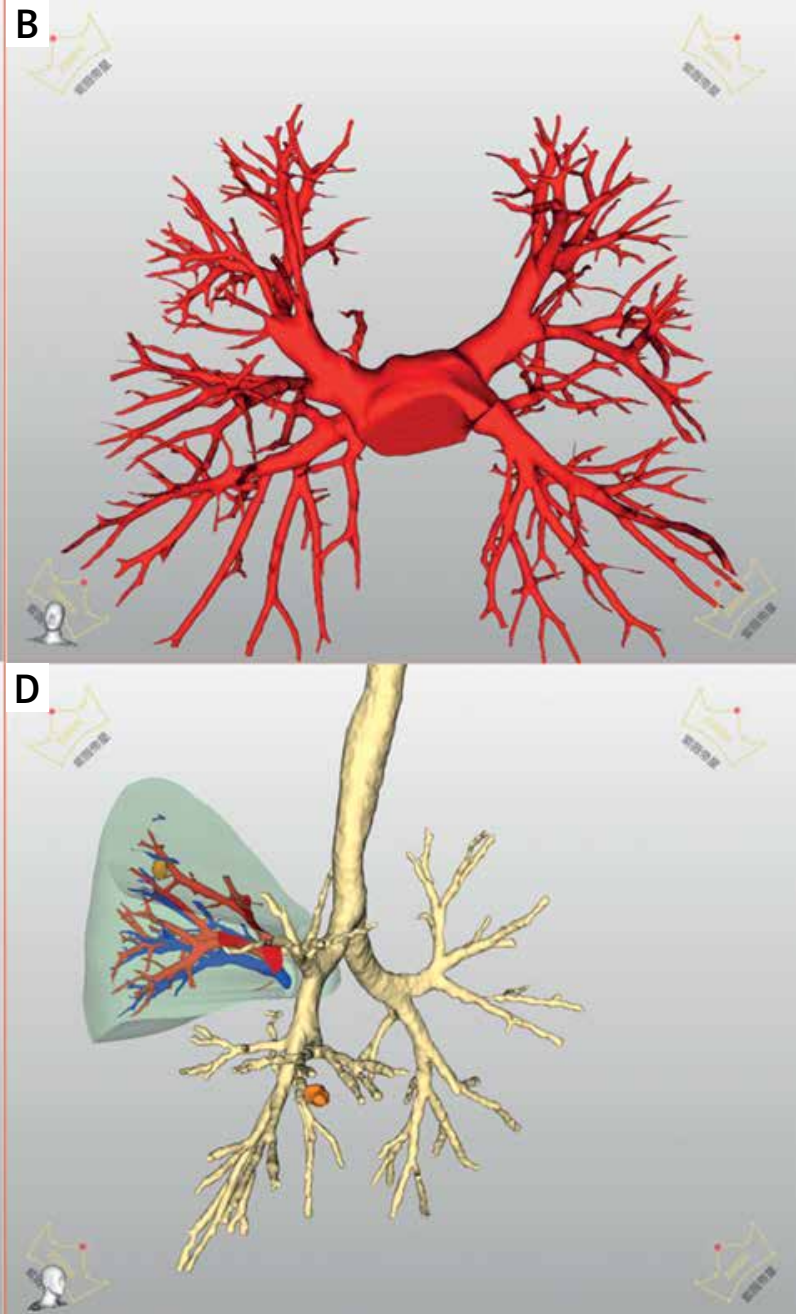


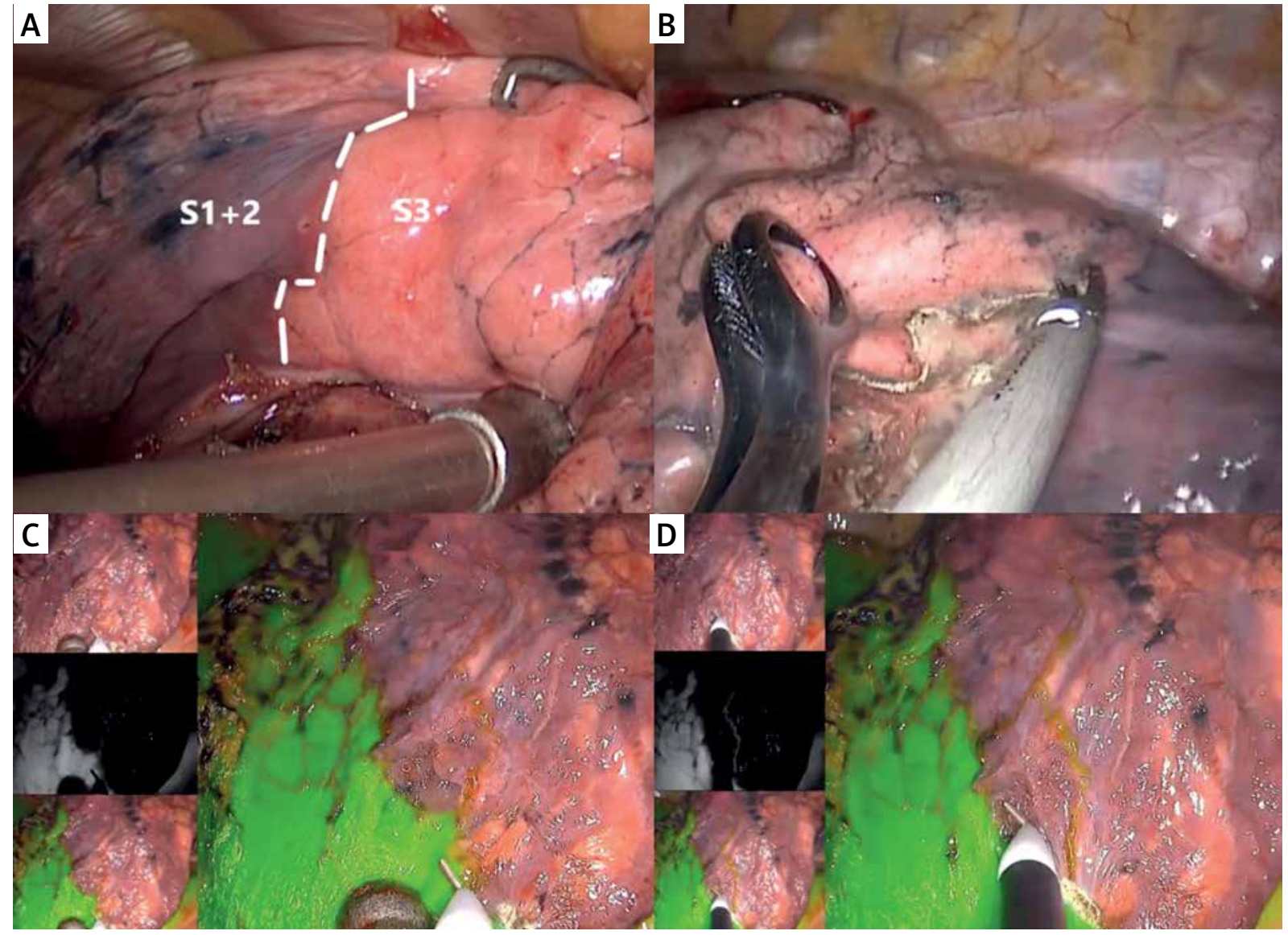

Photo 2. The method of confirming the boundary between lung segments. A, B - ventilation first and then collapse of lung. C, D - ICG was injected intravenously

between the two groups $(p>0.05)$. The prevalence of emphysema in the elderly group was significantly higher compared to that in the non-elderly group, and the difference between the two groups was statistically significant $(p=0.001)$. Moreover, the prevalence of hypertension and diabetes in the elderly group was also higher compared to that in the non-elderly group; the difference between the two groups was statistically significant $(p<0.05)$.

Table II shows the lung segments resected in the two groups of patients. In the elderly group, except for LS4, LS10, RS4, RS5, and RS10, all other lung segments were resected, including complex combined segmental resection of the lung for $\mathrm{LS} 8+9, \mathrm{LS} 9+10$, RS8+9, and RS9+10. The lung segment with the highest resection rate in the elderly group was LS6 (11 cases), RS6 (10 cases), and LS1+2 (10 cases). In the non-elderly group, resection of all segments of the lung except RS5 was performed, including complex combined segmental resection of the lung for
LS8+9, LS9+10, RS8+9, and RS9+10. Compared to the elderly group, the lung segments with the highest resection rate in this group were LS1+2 (22 cases), RS6 (16 cases), and LS6 (15 cases).

The perioperative observation indicators and related complications of the two groups are listed in Table III and statistically analyzed. Perioperative observation indicators include: surgery time, intraoperative blood loss, retention time of thoracic drainage tube, postoperative hospital stay, and patient satisfaction with the incision. There was no statistically significant difference in these observation indicators between the two groups $(p>0.05)$. It is noteworthy that uniportal video-assisted thoracoscopic segmental resection of the lung was performed for all patients in the two groups, without conversion to open surgery. The postoperative complications counted included pulmonary infection, alveolar leakage, arrhythmia, pulmonary embolism, incision infection, fever, and dyspnea, and the differences in 
Table I. Baseline characteristics data before operation

\begin{tabular}{|c|c|c|c|}
\hline Parameter & Elderly group $(n=63)$ & Non-elderly group $(n=112)$ & $P$-value \\
\hline Sex: & & & 0.506 \\
\hline Male & 28 & 44 & \\
\hline Female & 35 & 68 & \\
\hline Family history & 6 & 14 & 0.553 \\
\hline Smoking history & 13 & 24 & 0.875 \\
\hline Emphysema & 11 & 3 & $0.001^{a}$ \\
\hline Hypertension & 20 & 11 & 0.000 \\
\hline Diabetes & 14 & 10 & 0.014 \\
\hline Coronary heart disease & 6 & 4 & $0.103^{a}$ \\
\hline Occupation: & & & 0.743 \\
\hline Manual workers & 31 & 58 & \\
\hline Mental workers & 32 & 54 & \\
\hline BMI [kg/m²]: & & & 0.870 \\
\hline$<24$ & 34 & 59 & \\
\hline$\geq 24$ & 29 & 53 & \\
\hline Pathological type: & & & 0.830 \\
\hline Adenocarcinoma in situ & 21 & 40 & \\
\hline Microinvasive adenocarcinoma & 16 & 31 & \\
\hline Invasive adenocarcinoma & 26 & 41 & \\
\hline
\end{tabular}

aFisher's exact test was used instead, as there were expected cell frequencies < 5. Data are mean \pm SD or $n(\%)$.

Table II. Resected lung segments of the two groups

\begin{tabular}{|lccccc|}
\hline Left & Elderly group & Non-elderly group & Right & Elderly group & Non-elderly group \\
\hline LS1+2 & 10 & 22 & RS1 & 4 & 6 \\
\hline LS3 & 2 & 2 & RS2 & 5 & 12 \\
\hline LS4 & 0 & 2 & RS3 & 2 & 2 \\
\hline LS5 & 1 & 3 & RS4 & 0 & 2 \\
\hline LS6 & 11 & 15 & RS5 & 0 & 0 \\
\hline LS8 & 3 & 6 & RS6 & 10 & 16 \\
\hline LS9 & 1 & 2 & RS8 & 3 & 2 \\
\hline LS10 & 0 & 2 & RS9 & 1 & 2 \\
\hline LS8+9 & 2 & 4 & RS10 & 1 & 3 \\
\hline LS9+10 & 3 & 3 & RS8+9 & 3 & 3 \\
\hline
\end{tabular}

these complications between the two groups were not statistically significant $(p>0.05)$.

\section{Discussion}

Standard VATS lobectomy is performed with a greater risk for elderly patients with lung cancer who are often complicated with extrapulmonary underlying diseases, multiple organ dysfunction, and slow postoperative recovery, particularly for those with poor cardiopulmonary function, where the clinical application is limited $[6,7]$. In recent years, it has been reported that the sublobar resection in elderly patients with NSCLC can achieve similar effects as 
Table III. Postoperative complications and early surgical effect

\begin{tabular}{|c|c|c|c|}
\hline Parameter & Elderly group $(n=63)$ & Non-elderly group $(n=112)$ & $P$-value \\
\hline Surgical time [min] & $144.57 \pm 21.14$ & $153.34 \pm 18.65$ & 0.405 \\
\hline Blood loss [ml] & $119.18 \pm 53.57$ & $126.67 \pm 56.21$ & 0.367 \\
\hline Conversion to thoracotomy & 0 & 0 & \\
\hline Tube retention time [days] & $2.37 \pm 1.37$ & $2.12 \pm 1.42$ & 0.421 \\
\hline Hospital stay after surgery [days] & $3.75 \pm 1.40$ & $3.50 \pm 1.17$ & 0.752 \\
\hline Satisfaction with incision: & & & $0.786^{\mathrm{a}}$ \\
\hline Satisfactory & 59 & 106 & \\
\hline Unsatisfactory & 4 & 6 & \\
\hline \multicolumn{4}{|l|}{ Complications: } \\
\hline Pulmonary infection & 3 & 4 & $0.700^{a}$ \\
\hline Pulmonary air leakage & 5 & 8 & 0.848 \\
\hline Arrhythmia & 4 & 6 & 0.786 \\
\hline Pulmonary embolism & 1 & 0 & $0.360^{a}$ \\
\hline Incision infection & 0 & 0 & \\
\hline Fever & 7 & 13 & 0.865 \\
\hline Dyspnea & 2 & 3 & $0.851^{a}$ \\
\hline
\end{tabular}

aFisher's exact test was used instead, as there were expected cell frequencies $<5$. Data are mean \pm SD or $n(\%)$.

lobectomy [8]. Some researchers even proposed that the recent clinical efficacy of VATS sublobar resection in the treatment of elderly patients with NSCLC deserved greater acknowledgment, and recommended it as the clinically preferred surgical method $[9,10]$. Therefore, uniportal VATS segmental resection of the lung was performed for patients with lung cancer aged over 65 years with GGN on CT.

Uniportal VATS has the advantages of small trauma, esthetic wound, and only a single incision [5, 11], with the most commonly used being the intercostal incision. The intercostal incision is usually located between the anterior axillary line and midaxillary line, between the $4^{\text {th }}$ or $5^{\text {th }}$ intercostal space, with a length of about $3 \mathrm{~cm}$. The experience of our center is, when the incision is closer to the midaxillary line, it is more convenient for the surgeon to dissect the hilar structure and mediastinal lymph nodes; however, the linear cutting suture device is more difficult to place, making the surgical technique more complex for the surgeon. If the incision is more forward, the intercostal space will be slightly wider, farther from the hilum. Other thoracoscopic incisions have also been reported clinically. In 2017, Stamenovic et al. reported 20 cases of uniportal video-assisted thoracoscopic lobe resection and segmental resection of the lung from the back approach [12]. The incisions of these procedures were located at the $6^{\text {th }}$ intercostal "triangle of auscultation" of the back, with a length of $3.5-4.5 \mathrm{~cm}$. This incision was more beneficial for the exposure of the dorsal bronchus and blood vessels, it was easier to dissect the corresponding mediastinal lymph nodes, and the incision location was more hidden. In 2018, Shen et al. reported a case of uniportal video-assisted thoracoscopic right upper lobectomy through an axillary incision parallel to the skin fold and about $4 \mathrm{~cm}$ in length, which was highly esthetic [13]. Compared with the traditional surgery, uniportal VATS reduces the huge damage to the chest wall of elderly patients, effectively shortens the patient's postoperative recovery time, reduces the risk of complications, and improves the esthetics of traditional incisions [14-16]. In this study, a method with improved sutured incisions and a fixed thoracic drainage tube was used (Photo $3 \mathrm{~A}$ ), which made the surgical incision esthetic after removing the thoracic drainage tube (Photo $3 \mathrm{~B}$ ). The incision satisfaction of patients in both groups reached a high level, and there was no statistically significant difference between the two groups (93.7\% vs. 94.6\%, $p=0.786)$.

In uniportal video-assisted thoracoscopic segmental resection of the lung, it is difficult to master the technique due to the variation of anatomical structure of the lung segments among different in- 


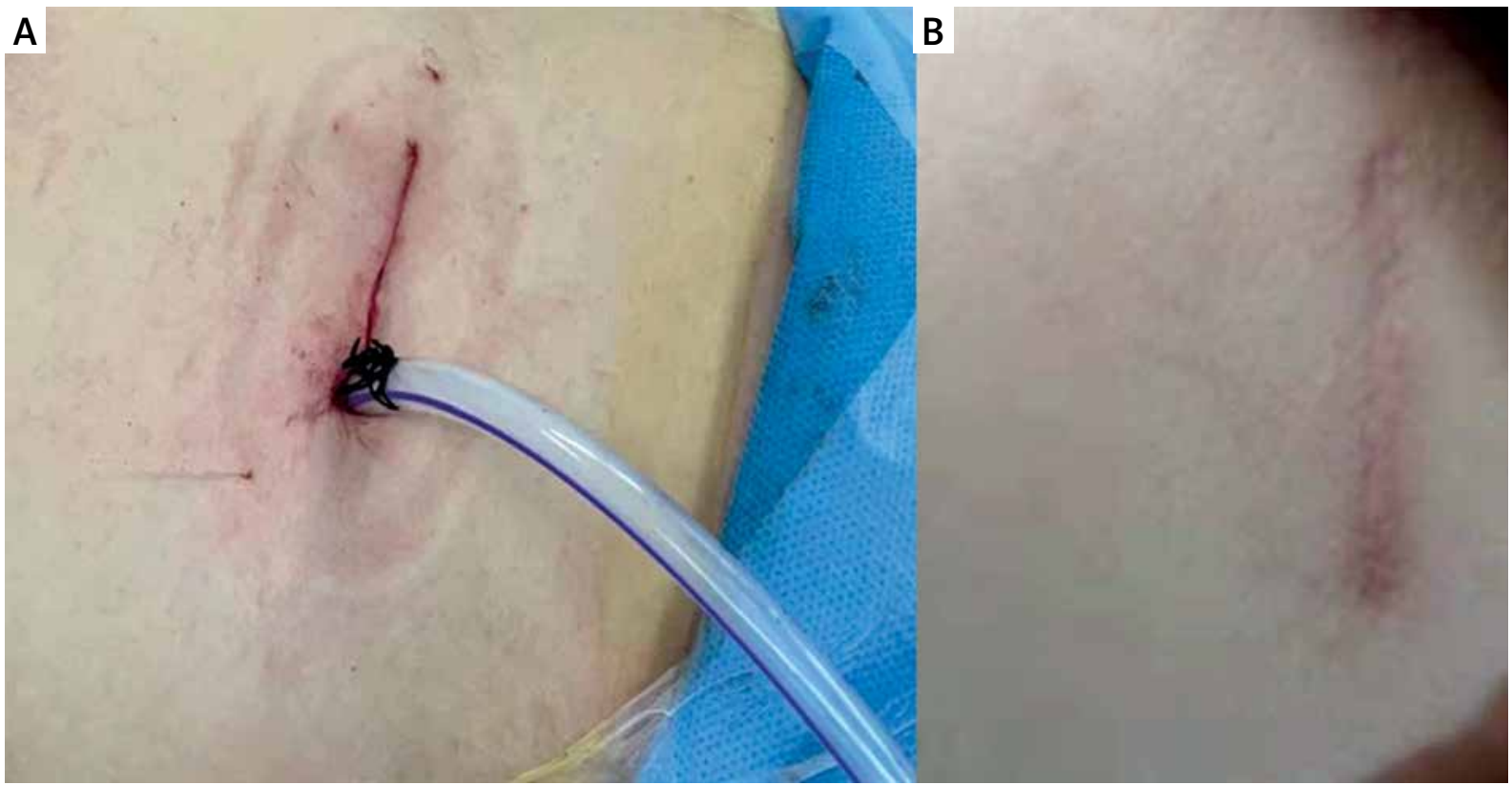

Photo 3. A novel method of suturing incision. A - After the operation, the incision was sutured with fishbone suture and the thoracic drainage tube was fixed. B - The wound healing of the patient was reexamined 3 months later

dividuals $[16,17]$. Due to the variation of the pulmonary vessels, beginners may transect the artery, adjacent to the lung segment, resulting in no blood supply to the lung tissue $[18,19]$. It is inaccurate to determine the intersegmental boundary by using the inflation-deflation method. If the pulmonary artery of the target pulmonary segment is not completely transected, the intersegmental boundary is also inaccurate, and the hilum cannot be lifted, causing some lung segment tissues, which should be retained, to be resected [20]. Moreover, if the pulmonary veins that are not part of the target lung segment are transected, it will cause venous return disorder, postoperative hemoptysis, and a second operation is required for lobectomy in severe cases [21]. If the pulmonary vein of the target lung segment is not completely transected, misjudgment will occur when judging the intersegmental boundary based on the intersegmental veins, and the lung tissue that should have been resected may be retained $[22,23]$. The experience is that preoperative MIMICS is used to convert 2D CT images into $3 \mathrm{D}$ images to accurately determine the vessels and bronchi of the target lung segment and guide the surgeon to perform the surgery.

In general, a simple inflation-deflation method is used to determine the intersegmental boundary of segmental resection of the lung [24]. However, elderly patients are often complicated with the presence of emphysema and lung hyperinflation, which makes it difficult to obtain the intermidsection boundary with this method $[25,26]$. In the study, 11 (17.5\%) patients in the elderly group suffered from emphysema, with a high incidence. For such patients, the near-infrared fluorescence guided thoracoscopic ICG reverse staining method was used to quickly and accurately confirm the intersegmental boundary. Moreover, this method can save time for the lung inflation and deflation, making the surgery process faster $[27,28]$. In this research center, the surgery time of the elderly group (144.57 \pm 21.14$)$ was slightly lower compared to that of the non-elderly group (153.34 \pm 18.65$)$; however, the difference was not statistically significant. This may be because although the near-infrared fluorescence guided thoracoscopic IGG reverse staining method was used for 11 patients with emphysema in the elderly group to determine the intersegmental boundary, the simple inflation-deflation method was used to determine the intermidsection boundary for the other 52 cases. After the intersegmental boundary is confirmed and marked, it is not easy to transect the lung segments precisely without affecting the inflation of the remaining lung tissue. On the basis of the 
experience, during the surgery, an ultrasonic knife is used to separate the originally 3D intersegmental structure into a plane along the intersegmental boundary. The high-frequency vibration energy of the ultrasonic knife can enable precise transecting and effective hemostasis synchronously, without eschar, thus ensuring the accuracy of the surgical field and deep surgery. Finally, the remaining plane is accurately processed by the linear transecting and suture device. This can not only ensure the precise resection of the target lung segment, but also make the wound clean, the remaining lung stretched and well inflated, and minimize the loss of lung function.

Early NSCLC patients with GGN often have no symptoms, and a large number are accidentally discovered during physical examination [29]. In addition, due to the large number of underlying diseases, it is difficult to distinguish the early lung cancer of elderly patients from their own chronic respiratory diseases, thus delaying the opportunity of treatment [30]. Some elderly patients recover slowly after surgery, and are prone to psychological characteristics such as anxiety and self-abandonment, which often become a major difficulty in treatment [31]. Moreover, with the increase of age, the internal organ function and immunity of elderly patients become poor, with complications of chronic diseases such as hypertension and diabetes before surgery [32, 33]. Surgical methods with longer postoperative recovery time and greater trauma will undoubtedly have a greater impact on patients' physiology and psychology [34, 35], while the application of uniportal video-assisted thoracoscopic segmental resection of the lung has advantages that do not exist in traditional thoracotomy: 1) Small surgical trauma, avoids damage to the ribs and muscles, reduces intraoperative blood loss and shortens postoperative recovery time, thereby avoiding a series of complications such as severe postoperative pain and poor wound healing due to large surgical wounds in elderly patients [36]; 2) Uniportal VATS has a clearer local exposure and a good visual angle, which is similar to the surgical field under the direct vision of thoracotomy. It can ensure the accuracy of the surgery, accurately identify the location of lesions and lymph lesions, and under the guidance of 3D images, can completely utilize the advantages of fine anatomy, maximize the remaining lung function of elderly patients, and improve the postoperative quality of life [37]; 3) The more esthetic wound makes the patient more sat- isfied with the prognosis, avoids the psychological and life disorders due to postoperative scar, is more conducive to the long-term recovery of the patients, and reduces the long-term impact of the surgery on the patient's mind and movement [38].

\section{Conclusions}

Uniportal video-assisted thoracoscopic segmental resection of the lung is feasible and safe in elderly patients with NSCLC aged over 65 years. Compared with non-elderly patients, the use of appropriate technology does not increase the difficulty and time of the surgery, or increase the postoperative complications, and is worthy of clinical promotion.

\section{Acknowledgments}

The authors thank International Science Editing (http://www.internationalscienceediting.com) for editing this manuscript.

\section{Conflict of interest}

The authors declare no conflict of interest.

\section{References}

1. Jemal A, Miller KD, Ma J, et al. Higher lung cancer incidence in young women than young men in the United States. N Engl J Med 2018; 378: 1999-2009.

2. Calado T, Antunes M, Cabral D, et al. Surgical treatment for elderly patients with lung cancer. Rev Port Cir Cardiotorac Vasc 2017; 24: 144

3. Hung WT, Hung MH, Wang ML, et al. Nonintubated thoracoscopic surgery for lung tumor: seven years' experience with 1,025 patients. Ann Thorac Surg 2019; 107: 1607-12.

4. Bulgarelli Maqueda L, García-Pérez A, Minasyan A, et al. Uniportal VATS for non-small cell lung cancer. Gen Thorac Cardiovasc Surg 2020; 68: 707-15.

5. Sihoe AD. Uniportal video-assisted thoracic (VATS) lobectomy. Ann Cardiothorac Surg 2016; 5: 133-44.

6. Agostini PJ, Lugg ST, Adams K, et al. Risk factors and short-term outcomes of postoperative pulmonary complications after VATS lobectomy. J Cardiothorac Surg 2018; 13: 28.

7. McDonald F, De Waele M, Hendriks LE, et al. Management of stage I and II nonsmall cell lung cancer. Eur Respir J 2017; 49: 1600764.

8. Yang F, Sui X, Chen $X$, et al. Sublobar resection versus lobectomy in surgical treatment of elderly patients with early-stage non-small cell lung cancer (STEPS): study protocol for a randomized controlled trial. Trials 2016; 17: 191.

9. Wang $\mathrm{HH}$, Zhang CZ, Zhang BL, et al. Sublobar resection is associated with improved outcomes over radiotherapy in the management of high-risk elderly patients with Stage I non- 
small cell lung cancer: a systematic review and meta-analysis. Oncotarget 2017; 8: 6033-42.

10. Zhang Z, Feng $\mathrm{H}$, Zhao H, et al. Sublobar resection is associated with better perioperative outcomes in elderly patients with clinical stage I non-small cell lung cancer: a multicenter retrospective cohort study. J Thorac Dis 2019; 11: 1838-48.

11. Galvez C, Sesma J, Bolufer S, et al. The techniques of uniportal video-assisted thoracoscopic surgery: lower lobectomies and lymphadenectomy. J Thorac Dis 2019; 11 (Suppl 16): S2095-107.

12. Stamenovic D, Bostanci K, Messerschmidt A. Posterior uniportal video-assisted thoracoscopic surgery for anatomical lung resections. J Thorac Dis 2017; 9: 5261-6.

13. Shen Y, Zhang Y, Sun J, et al. Transaxillary uniportal video assisted thoracoscopic surgery for right upper lobectomy. J Thorac Dis 2018; 10: E214-7.

14. Bin Yameen TA, Gupta V, Behzadi A. Uniportal versus multiportal video-assisted thoracoscopic surgery in the treatment of lung cancer: a Canadian single-centre retrospective study. Can J Surg 2019; 62: 468-74.

15. Hernandez-Arenas LA, Purmessur RD, Gonzalez-Rivas D. Uniportal video-assisted thoracoscopic segmentectomy. I Thorac Dis 2018; 10 (Suppl 10): S1205-14.

16. Kim HK, Han KN. Uniportal video-assisted thoracoscopic surgery segmentectomy. Thorac Surg Clin 2017; 27: 387-98.

17. Stamenovic D, Messerschmidt A. Uniportal video-assisted thoracoscopic surgery for anatomical fissureless anterior segment resection (S3) of the right upper lobe followed by lymph node dissection. Multimed Man Cardiothorac Surg 2018; 2018. doi: 10.1510/mmcts.2018.001.

18. Amore D, Imitazione P, Palma A, et al. Conversion to thoracotomy during VATS segmentectomy for treatment of symptomatic endobronchial hamartoma. Int J Surg Case Rep 2018; 51: 272-4.

19. Andolfi M, Potenza R, Seguin-Givelet A, et al. Identification of the intersegmental plane during thoracoscopic segmentectomy: state of the art. Interact Cardiovasc Thorac Surg 2020; 30: 329-36.

20. Ikeda T, Nakano J. Anormalous systemic arterial supply to the basal segments of the lung presented with chest back pain: report of a case. Kyobu Geka 2018; 71: 472-5.

21. Matsuura Y, Mun M, Ichinose J, et al. Recent fluorescence-based optical imaging for video-assisted thoracoscopic surgery segmentectomy. Ann Transl Med 2019; 7: 32.

22. Nakazawa S, Shimizu K, Mogi A, et al. VATS segmentectomy: past, present, and future. Gen Thorac Cardiovasc Surg 2018; 66: 81-90.

23. Pan X, Zhang Y, Ren S, et al. Video-assisted thoracoscopic superior segmentectomy of the right lower lobe. J Thorac Dis 2016; 8: 1349-52.

24. Sun Y, Zhang Q, Wang Z, et al. Is the near-infrared fluorescence imaging with intravenous indocyanine green method for identifying the intersegmental plane concordant with the modified inflation-deflation method in lung segmentectomy? Thorac Cancer 2019; 10: 2013-21.

25. Saito D, Matsumoto I, Waseda R, et al. A method to identify pulmonary intersegmental planes with intravenous vitamin B2 injection. Surg Today 2021; 51: 836-43.

26. Vallurupalli S, Kasula S, Kumar Agarwal S, et al. A novel stent inflation protocol improves long-term outcomes compared with rapid inflation/deflation deployment method. Catheter Cardiovasc Interv 2017; 90: 233-40.

27. Uramoto H, Motono N. ICG easily detects not only the segmental plane, but also the course and blood distribution of the bronchial artery"case report". Ann Med Surg 2018; 28: 28-9.

28. Zhang C, Lin H, Fu R, et al. Application of indocyanine green fluorescence for precision sublobar resection. Thorac Cancer 2019; 10: 624-30.

29. Osarogiagbon RU, Veronesi G, Fang W, et al. Early-stage NSCLC: advances in thoracic oncology 2018. I Thorac Oncol 2019; 14: 968-78.

30. Singh A, Kant R, Saluja TS, et al. Differential diagnosis of nonsmall cell lung carcinoma by circulating microRNA. J Cancer Res Ther 2020; 16: 127-31.

31. Ambrogi V, Tajè R, Mineo TC. Nonintubated video-assisted wedge resections in peripheral lung cancer. Thorac Surg Clin 2020; 30: 49-59

32. Detillon D, Aarts MJ, De Jaeger K, et al. Video-assisted thoracic lobectomy versus stereotactic body radiotherapy for stage I nonsmall cell lung cancer in elderly patients: a propensity matched comparative analysis. Eur Respir J 2019; 53: 1801561.

33. Igai $H$, Kamiyoshihara $M$, Ibe $T$, et al. Surgical treatment for elderly patients with secondary spontaneous pneumothorax. Gen Thorac Cardiovasc Surg 2016; 64: 267-72.

34. Sastre I, España M, Ceballos R, et al. Video-assisted thoracoscopic apicoposterior segmentectomy. Multimed Man Cardiothorac Surg 2020; 2020. doi: 10.1510/mmcts.2020.037.

35. Stamenovic D, Messerschmidt A, Schneider T. Surgery for lung tumors in the elderly: a retrospective cohort study on the influence of advanced age (over 80 years) on the development of complications by using a multivariate risk model. Int I Surg 2018; 52: 141-8.

36. Li J, Qiu B, Scarci M, et al. Uniportal video-assisted thoracic surgery could reduce postoperative thorax drainage for lung cancer patients. Thorac Cancer 2019; 10: 1334-9.

37. Takahashi Y, Miyajima M, Tada M, et al. Outcomes of completion lobectomy long after segmentectomy. J Cardiothorac Surg 2019; 14: 116.

38. Ye B, Wang M. Video-assisted thoracoscopic surgery versus thoracotomy for non-small cell lung cancer: a meta-analysis. Comb Chem High Throughput Screen 2019; 22: 187-93.

Received: 2.05.2021, accepted: 14.05.2021. 